\title{
A novel splicing mutation in SLC9A6 in a boy with Christianson syndrome
}

\author{
Daisuke leda', Ikumi Hori ${ }^{1}$ ', Yuji Nakamura', Kei Ohashi', Yutaka Negishi', Ayako Hattori', Atsuko Arisaka², \\ Setsuko Hasegawa ${ }^{2,3}$ and Shinji Saitoh (1)
}

\begin{abstract}
A loss of function mutation in SLC9A6 (Xq26.3) is responsible for Christianson syndrome in males. We identified a novel splicing mutation (NM_006359.2:C.1141-8C>A) of SLC9A6 in a seven-year-old boy with microcephaly, severe developmental delay, and intractable epilepsy. Functional analysis found multiple aberrant transcripts, none of which maintained the canonical open reading frame. Computer prediction tools, however, failed to detect all of the aberrant transcripts.
\end{abstract}

A loss of function mutation in the SLC9A6 gene (Xq26.3) is responsible for Christianson syndrome (CS), which is characterized by severe global developmental delay, developmental regression, acquired microcephaly, intractable epilepsy, ataxia, ophthalmoplegia, and sometimes, death at a young age $\mathrm{e}^{1,2}$. The clinical features of CS overlap with those of Angelman syndrome (AS), which is caused by a lack of expression of the maternally inherited $U B E 3 A$ gene located on $15 \mathrm{q} 11.2^{3}$. SLC9A6 encodes the $\mathrm{Na}+/ \mathrm{H}+$ exchanger protein NHE6. This protein regulates the luminal $\mathrm{pH}$ of early and recycling endosomes involved in the trafficking of proteins essential for structural and functional plasticity at glutamatergic synapses ${ }^{4}$. NHE6 has an important role in the growth of dendritic spines and the development of normal brain wiring ${ }^{5}$. Here, we identified a novel SLC9A6 splicing mutation in a sevenyear-old boy with microcephaly, severe developmental delay, and intractable epilepsy. To evaluate the mutation, we used various computer prediction tools as well as reverse transcription polymerase chain reaction (RT-PCR) and cloning to assess transcripts and confirm the pathogenicity of the mutation.

\footnotetext{
Correspondence: Shinji Saitoh (ss11@med.nagoya-cu.ac.jp)

'Department of Pediatrics and Neonatology, Nagoya City University Graduate School of Medical Sciences, Nagoya, Japan

${ }^{2}$ Department of Pediatrics, Tokyo-Kita Medical Center, Tokyo, Japan

Full list of author information is available at the end of the article.
}

The case study, a seven-year-old Japanese boy, was born at term with a birth weight of $2978 \mathrm{~g}(-0.4 \mathrm{SD})$, length of $50.2 \mathrm{~cm}(+0.4 \mathrm{SD})$, and head circumference of $31.4 \mathrm{~cm}$ $(-1.5 \mathrm{SD})$. His development delayed gradually, achieving head control at four months, sitting at nine months, and pulling to stand at two years. Currently, he cannot stand independently nor speak meaningful words. At 10 months of age, he developed intractable seizures of variable types: tonic-clonic convulsion, impairment of consciousness, focal seizure, and epileptic negative myoclonus. He was treated with multiple antiepileptic drugs that had insufficient therapeutic effects. An electroencephalography (EEG) performed at four years showed focal epileptic discharges with generalization in multiple foci (Fig. 1a). At four years of age, his weight was $14.5 \mathrm{~kg}(-0.7 \mathrm{SD})$, length was $102 \mathrm{~cm}(-0.1 \mathrm{SD})$, and head circumference was $46.2 \mathrm{~cm}(-2.6 \mathrm{SD})$, indicating microcephaly. Brain magnetic resonance imaging (MRI) (performed at 1,2 , and 4 years of age) and magnetic resonance spectroscopy (MRS) (performed at 4 years of age) showed no abnormal findings (Fig. 1b, c). At five years of age, we suspected the diagnosis of AS due to severe developmental delay, trunk ataxia, intractable seizures, microcephaly, and frequent smiling.

We first performed genetic tests, including fluorescent in situ hybridization (FISH), DNA methylation, and mutation analysis of the protein coding exons of $U B E 3 A$ 


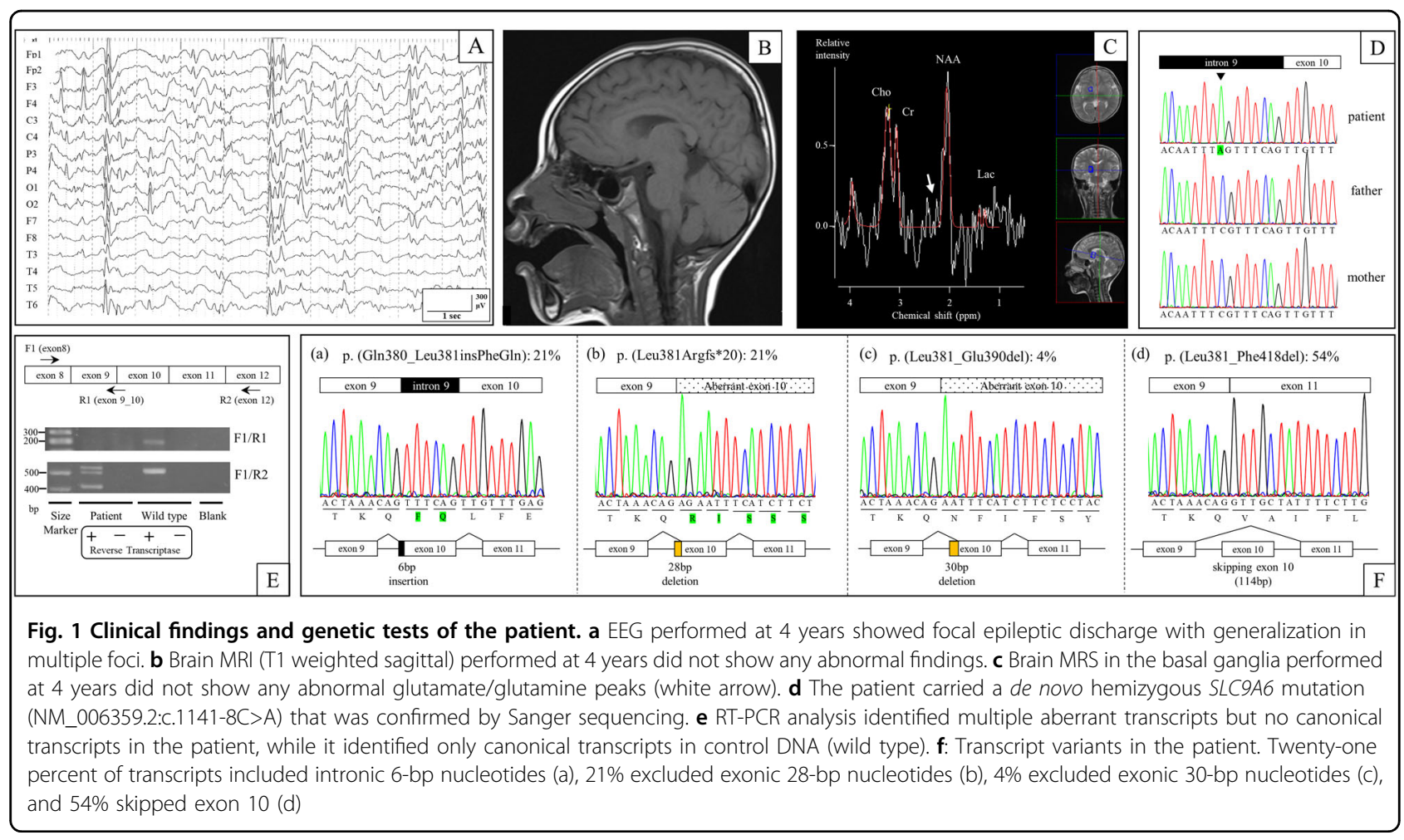

by Sanger sequencing, but all of the tests were normal. Next, we performed targeted next-generation sequencing with the Ion Torrent Personal Genome Machine system (Life Technologies, Carlsbad, California). An amplicon library of the target exons and flanking sequence was prepared with the use of an Ion AmpliSeq Custom Panel (Life Technologies), which included UBE3A, SLC9A6, TCF4, MBD5, CDKL5, MECP2, and FOXG1. Sequence analysis pipelines were established with use of the workflow in CLC Genomic Workbench 7.0 (CLC bio, Aarhus, Denmark). We identified a de novo hemizygous splicing mutation (c.1141-8C>A) in SLC9A6 (NM_006359.2), which was confirmed by Sanger sequencing using the SLC9A6-intron 9-Fwd (5'-TCCACATTTGCTCCCTTC T-3') and SLC9A6-exon 10-Rev (5'-ACCACATACTCA AAACCCAC-3') primer pair (Fig. 1d). We predicted that the mutation affected RNA splicing because it resulted in a new AG acceptor site six nucleotides upstream of the canonical acceptor site of exon 10.

To evaluate the mutation, we used multiple computer prediction tools. CRYP-SKIP (http://cryp-skip.img.cas.cz/) provides an overall probability of cryptic splice-site activation (as opposed to exon skipping) termed $P_{\text {CR-E }}{ }^{6} \cdot P_{\text {CR-E }}$ calculates a value between 0 and 1 , and lower values favor exon skipping. The $P_{\text {CR-E }}$ prediction score for SLC9A6 (c.1141-8C >A) was 0.20, thus favoring exon skipping. Next, we used Alamut Visual software (version 2.10, Interactive Biosoftware, Rouen, France), which assesses genomic sequences (wild type and mutant) using five splicing prediction tools (SpliceSiteFinder-like, MaxEntScan, Neural Network Splice, GeneSplicer, and Human Splicing Finder) based on different algorithms ${ }^{7}$. All five algorithms predicted a strength reduction in the canonical acceptor site. The prediction scores for the aberrant acceptor site (six nucleotides upstream of the canonical acceptor site) increased with three algorithms, and the scores did not change at any point downstream of the canonical acceptor site (Table 1).

To confirm the RNA splicing results, we performed RTPCR, cloning, and Sanger sequencing using total RNA from Epstein-Barr virus-induced lymphoblastoid cell lines established from peripheral leukocytes. RT-PCR using the SLC9A6-exon 8-Fwd1 (5'-ACCAAATTACGGGAGTTC CA-3') and SLC9A6-exon 12-Rev2 (5'-CACCACCAA ATACCCACAC- $3^{\prime}$ ) primer pair revealed the presence of multiple transcripts (Fig. 1e). SLC9A6 cDNA was then ligated into a TOPO cloning vector (Life Technologies) and transformed into TOP10 Competent Cells (Life Technologies), and 24 colonies were screened by extracting plasmid DNA using a QIAprep Spin Miniprep Kit (Qiagen, Hilden, Germany). Sanger sequencing of the plasmid clones identified four unique, aberrant transcripts but no canonical transcripts. Of the 24 cDNA transcripts screened, five (21\%) had the six-nucleotide addition of the intronic sequence to the 5 ' end of exon 10 that was predicted by our in silico analysis (Fig. 1f, transcript (a)). We also identified five (21\%) transcripts and one (4\%) transcript with 5' exon 10 deletions of 28 and 30 nucleotides, 
Table 1 In silico and functional splicing analysis from five prediction algorithms and in vitro RT-PCR analysis of mRNA transcripts

\begin{tabular}{|c|c|c|c|c|c|c|c|c|c|c|c|}
\hline \multirow[t]{2}{*}{ cDNA position ${ }^{\mathrm{a}}$} & \multicolumn{2}{|c|}{ SSF $(0-100)$} & \multicolumn{2}{|c|}{$\begin{array}{l}\text { MaxEntScan } \\
(0-16)\end{array}$} & \multicolumn{2}{|c|}{$\begin{array}{l}\text { NNSPLICE } \\
(0-1)\end{array}$} & \multicolumn{2}{|c|}{$\begin{array}{l}\text { GeneSplicer } \\
(0-21)\end{array}$} & \multicolumn{2}{|c|}{ HSF $(0-100)$} & \multirow[t]{2}{*}{ In vitro observed mRNA transcripts } \\
\hline & WT & MUT & WT & MUT & WT & MUT & WT & MUT & WT & MUT & \\
\hline \multicolumn{12}{|c|}{ Canonical splice site } \\
\hline c. 1141 & 82.52 & $\mathrm{NE}$ & 8.27 & 1.58 & 0.74 & $\mathrm{NE}$ & 2.77 & $\mathrm{NE}$ & 88.42 & 85.82 & $0 \%$ \\
\hline \multicolumn{12}{|l|}{ Cryptic splice site } \\
\hline c.1141-6 & $N E$ & 76.86 & $\mathrm{NE}$ & 5.30 & $N E$ & $\mathrm{NE}$ & NE & $N E$ & $N E$ & 78.15 & $21 \%$ \\
\hline c. 1150 & $\mathrm{NE}$ & $\mathrm{NE}$ & $\mathrm{NE}$ & $N E$ & $N E$ & $\mathrm{NE}$ & $N E$ & $\mathrm{NE}$ & 72.75 & 72.75 & $0 \%$ \\
\hline c.1169 & 83.44 & 83.44 & 6.12 & 6.12 & 0.64 & 0.64 & $\mathrm{NE}$ & $\mathrm{NE}$ & 87.60 & 87.60 & $21 \%$ \\
\hline c.1171 & 72.43 & 72.43 & $\mathrm{NE}$ & $N E$ & $\mathrm{NE}$ & $\mathrm{NE}$ & NE & $\mathrm{NE}$ & 76.93 & 76.93 & $4 \%$ \\
\hline c.1216 & 88.37 & 88.37 & 8.92 & 8.92 & 0.67 & 0.67 & NE & $\mathrm{NE}$ & 91.52 & 91.52 & $0 \%$ \\
\hline
\end{tabular}

SSF Splice Site Finder-like, HSF Human Splicing Finder, WT wild type, MUT mutant, NE not evaluated

${ }^{a}$ First nucleotide of the acceptor splice site

respectively, as well as 13 transcripts (54\%) with complete skipping of exon 10 (Fig. 1f, transcripts (b), (c), and (d)). Furthermore, we performed RT-PCR using the SLC9A6exon 8-Fwd1 and SLC9A6-exon9_10-Rev1 (5'GCTCAAACAACTGTTTAGTTCTA-3') primer pair, which amplified only canonical transcripts, and it revealed canonical transcripts in control DNA but no amplification in that of the patient.

CS was first reported in 1999 in a Caucasian South African family with multiple affected males presenting with severe intellectual disability, mutism despite apparently normal hearing, intractable epilepsy, and limited life expectancy ${ }^{1}$. As some of the clinical features of CS are shared with AS, 1.8-5.5\% patients with AS-like phenotypes have SLC9A6 mutations ${ }^{3,8}$. The characteristic features that distinguish CS from AS are external ophthalmoplegia, developmental regression with loss of motor skills, progressive atrophy of the inferior cerebellar vermis, and an increased glutamine-glutamate peak in the basal ganglia on $\mathrm{MRS}^{9}$. Our patient, however, did not show any of these characteristic features at seven years of age. Pescosolido et al. reported that CS patients had regression in walking (57\%), eating (14\%), loss of few words/sounds (57\%), eye contact/facial expressions (14\%) and other fine/gross motor skills (14\%) after a medical illness and/or seizure cluster ${ }^{10}$; therefore, we intend to follow our patient carefully.

Using target sequencing, we identified a de novo hemizygous intronic mutation (c.1141-8C $>\mathrm{A}$ ) in SLC9A6 (NM_006359.2), which resulted in a new AG acceptor site six nucleotides upstream of the canonical acceptor site of exon 10. In silico computer prediction analysis was performed prior to functional analysis of the mutation. CRYP-SKIP predicted the mutation would tend to cause exon skipping. Alamut visual predicted a decreased score for the canonical acceptor site of exon 10 in all five algorithms and an increased score for c.1141-6, which is adjacent to the aberrant AG acceptor site, in 3 of the 5 algorithms. To confirm the differential RNA splicing caused by the intronic mutation, we performed functional analyses using RT-PCR, cloning, and Sanger sequencing. We found multiple aberrant transcripts in SLC9A6 involving exon 10, but no canonical transcripts were identified. Twenty-one percent of transcripts had the sixnucleotide addition of the intronic sequence to the $5^{\prime}$-end of exon 10, as predicted by our in silico analysis, which leads to a two-amino-acid insertion (p.(Gln380 Leu381insPheGln)) that we termed transcript (a). Transcripts (b) and (c) had $5^{\prime}$ exon 10 deletions of 28 and 30 nucleotides occurring in 21 and $4 \%$ of transcripts, respectively, leading to a p.(Leu381Argfs*20) frameshift in transcript (b) and a 10-amino-acid deletion (p.(Leu381_Glu390del)) in transcript (c). Fifty-four percent of transcripts had complete skipping of exon 10, termed transcript (d), due to a 114-bp deletion that led to a 38amino-acid deletion (p.(Leu381_Phe418del)). Exon 10 in SLC9A6 encodes part of the functional domain that interacts with angiotensin II type 2 receptor ${ }^{11}$. Transcripts (b) and (d) (frameshift mutation and single exon deletion, respectively) are likely to disrupt gene function. The functional consequences of transcripts (a) and (c) (small in-frame insertion/deletion) are unclear as they are also located in the functional domain and only correspond to $25 \%$ of transcripts. Since the phenotype of the patient is consistent with CS, we conclude that not enough functional transcripts of SLC $9 A 6$ are being expressed, and the c.1141-8C >A mutation is pathogenic.

Comparing computer predictions to RNA transcript analysis, transcripts (a) and (d) were predicted by Alamut 
visual and CRYP-SKIP, respectively, but transcripts (b) and (c) were not predicted. Previous studies comparing the functional consequences of splice site mutations in $H R$ (using CRYP-SKIP) ${ }^{12}$ and MYBPC3, ACTC1, and $S C N 5 A$ (using Alamut analysis) ${ }^{7}$ concluded that prediction programs underestimate the impact of intronic mutations and that functional analyses, such as RT-PCR and minigene analysis, are necessary. In our experience, computer prediction tools predicted two of the four aberrant transcripts detected by RT-PCR, highlighting the need to develop more accurate computer prediction tools.

\section{HGV database}

The relevant data from this Data Report are hosted at the Human Genome Variation Database at https://doi.org/10.6084/m9.figshare.hgv.2543

\section{Acknowledgements}

We thank the patient and family who participated in our study.

\section{Author details}

${ }^{1}$ Department of Pediatrics and Neonatology, Nagoya City University Graduate School of Medical Sciences, Nagoya, Japan. ²Department of Pediatrics, TokyoKita Medical Center, Tokyo, Japan. ${ }^{3}$ Department of Pediatrics and Developmental Biology, Graduate School of Medical and Dental Sciences, Tokyo Medical and Dental University, Tokyo, Japan

\section{Conflict of interest}

The authors declare that they have no conflict of interest.

\section{Publisher's note}

Springer Nature remains neutral with regard to jurisdictional claims in published maps and institutional affiliations.
Received: 6 August 2018 Revised: 10 January 2019 Accepted: 18 February 2019.

Published online: 25 March 2019

\section{References}

1. Christianson, A. L. et al. X linked severe mental retardation, craniofacial dysmorphology, epilepsy, ophthalmoplegia, and cerebellar atrophy in a large South African kindred is localised to Xq24-q27. J. Med. Genet. 36, 759-766 (1999).

2. Schroer, R. J. et al. Natural history of Christianson syndrome. Am. J. Med. Genet. A. 152A, 2775-2783 (2010).

3. Gilfillan, G. D. et al. SLC9A6 mutations cause X-linked mental retardation, microcephaly, epilepsy, and ataxia, a phenotype mimicking Angelman syndrome. Am. J. Hum. Genet. 82, 1003-1010 (2008).

4. Deane, E. C. et al. Enhanced recruitment of endosomal $\mathrm{Na}+/ \mathrm{H}+$ exchanger NHE6 into Dendritic spines of hippocampal pyramidal neurons during NMDA receptor-dependent long-term potentiation. J. Neurosci. 33, 595-610 (2013).

5. Takahashi, Y. et al. A loss-of-function mutation in the SLC9A6 gene causes $X$-linked mental retardation resembling Angelman syndrome. Am. J. Med. Genet. B. Neuropsychiatr. Genet. 156B, 799-807 (2011).

6. Divina, P., Kvitkovicova, A., Buratti, E. \& Vorechovsky, I. Ab initio prediction of mutation-induced cryptic splice-site activation and exon skipping. Eur. J. Hum. Genet. 17, 759-765 (2009).

7. Frisso, G. et al. Functional studies and in silico analyses to evaluate non-coding variants in inherited cardiomyopathies. Int. J. Mol. Sci. 17, 1883 (2016).

8. Fichou, Y. et al. Mutation in the SLC9A6 gene is not a frequent cause of sporadic Angelman-like syndrome. Eur. J. Hum. Genet. 17, 1378-1380 (2009).

9. Tan, W.-H., Bird, L. M., Thibert, R. L. \& Williams, C. A. If not Angelman, what is it? a review of Angelman-like syndromes. Am. J. Med. Genet. A. 164A, 975-992 (2014).

10. Pescosolido, M. F. et al. Genetic and phenotypic diversity of NHE6 mutations in Christianson syndrome. Ann. Neurol. 76, 581-593 (2014).

11. Zanni, G. et al. A novel mutation in the endosomal $\mathrm{Na}+/ \mathrm{H}+$ exchanger NHE6 (SLC9A6) causes Christianson syndrome with electrical status epilepticus during slow-wave sleep (ESES). Epilepsy Res. 108, 811-815 (2014).

12. Refke, M. et al. Functional analysis of splice site mutations in the human hairless (HR) gene using a minigene assay. Br. J. Dermatol. 165, 1127-1132 (2011). 